\title{
Underlying Event Studies at H1
}

\author{
Lluís Martí Magro on behalf of the H1 Collaboration. \\ Universidad Autónoma de Madrid - Dept de Físca Teórica \\ Módulo C-XI, Facultad de Ciencias, Cantoblanco, 28049 Madrid - Spain
}

\begin{abstract}
In lepton-hadron collisions an almost real photon interacts as a point-like (direct) particle as well as a composite hadron-like (resolved) system. Event samples with enriched direct- or resolved-photon events are selected by measuring the photon energy fraction entering in the hard scattering, $x_{\gamma}^{o b s}$. This allows the study of the Underlying Event (UE) and Multiple Parton Interactions (MPI) with a new strategy not possible at hadron colliders. The $\mathrm{H} 1$ collaboration studied photoproduction events with at least two jets with $P_{T}^{\text {jet }}>5 \mathrm{GeV}$ each. The highest transverse momentum jet (leading jet) defines four regions in azimuth: the toward region, defined by the leading jet, the away region, in the opposite hemisphere and two transverse regions between them. In each region, the charged particle multiplicity is measured and compared to models.
\end{abstract}

\section{Introduction}

In $e p$ collisions at HERA the mediator boson is a virtual photon. If the virtuality is high the photon interacts as a point-like particle (direct). At low virtualities the photon may fluctuate into a quark-antiquark pair and develop an even more complicated hadronic structure (resolved). In this case, a parton from the photon interacts with a parton from the proton and only a fraction of the energy from the resolved photon enters in the hard scattering. At HERA, these events can be selected by measuring the photon energy fraction entering in the hard scattering, $x_{\gamma}^{\text {obs }}$, by measuring the transverse momentum and pseudo-rapidity of the two leading jets:

$$
x_{\gamma}^{o b s}=\frac{P_{T}^{j e t 1} e^{-\eta^{j e t 1}}+P_{T}^{j e t 2} e^{-\eta^{j e t 2}}}{2 E_{\gamma}},
$$

Monte Carlo programs (MC) simulate ep collisions with a 2-to-2 parton scattering in leading order $\alpha_{s}$. For direct photoproduction, $x_{\gamma}^{\text {obs }}>0.7$, bosongluon fusion is the most important contribution to dijet production. In the event generation, initial and final state parton radiation and the contributions from the proton remnant are simulated. Hadronisation models are applied to produce colourless particles. In this picture, the primary two hard partons lead to two jets while the other parton emissions constitute the underlying event (UE).

Remnant-remnant interactions are only present when both interacting particles have a composite structure. This can happen for resolved photon

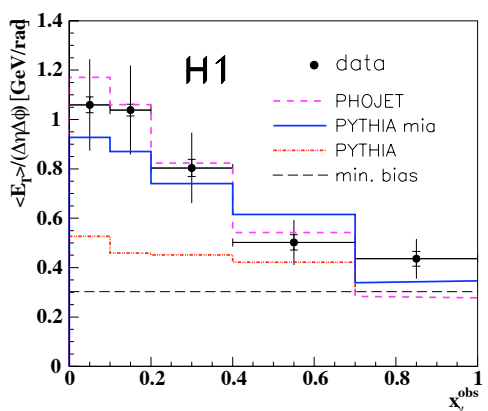

Figure 1: Average transverse energy density. events, $x_{\gamma}^{o b s}<0.7$, via Multiple Parton Interactions (MPI). By definition, these MPI are part of the UE. Therefore, selecting events with direct (resolved) photons excludes (allows) MPI 
from the UE. This is an advantage of a lepton-hadron collider compared to a hadron-hadron collider.

At $\mathrm{H} 1$ the structure of the photon and the effect of MPI had been studied [1] by measuring the energy flow outside jets in photoproduction events, figure 1. Although PYTHIA without MPI predicts a higher transverse energy distribution at lower $x_{\gamma}^{o b s}$, it is clearly not enough to describe the data. Only when including MPI (here PYTHIA mia) between the remnants the data can be described.

The description of MPI in particular and in general of the UE is very important for LHC physics: Higgs searches and multi-jet analyses like for the top quark require a proper description of the underlying QCD aspects. Different MPI models and parton dynamics approaches, however, give very different predictions at higher energies [2]. The strategy presented here consists of separating the point-like from the resolved contributions, i.e. events with only one remnant from those with two remnants where MPI are possible. Compared to hadron colliders, MPI is expected to be better separated from the rest of the UE (parton dynamics, hadronisation, etc).

\section{Charged particle multiplicity in photoproduction}

MPI and its contribution to the UE were studied by the H1 collaboration [3, 4] using dijet photoproduction. Events with photon virtuality $Q^{2}<0.01 \mathrm{GeV}^{2}$ and inelasticity $0.3<y<0.65$ were selected. The jets were defined applying the inclusive $k_{t}$-jet cluster algorithm [5] in the laboratory frame. The jets were required to have transverse momentum $P_{T}^{\text {jet }}>5 \mathrm{GeV}$ and pseudo-rapidity $\left|\eta^{\text {jet }}\right|<1.5$. Within these events, charged particles with transverse momenta $P_{T}^{\text {track }}>150 \mathrm{MeV}$ in the range $\left|\eta^{\text {track }}\right|<1.5$ were selected.

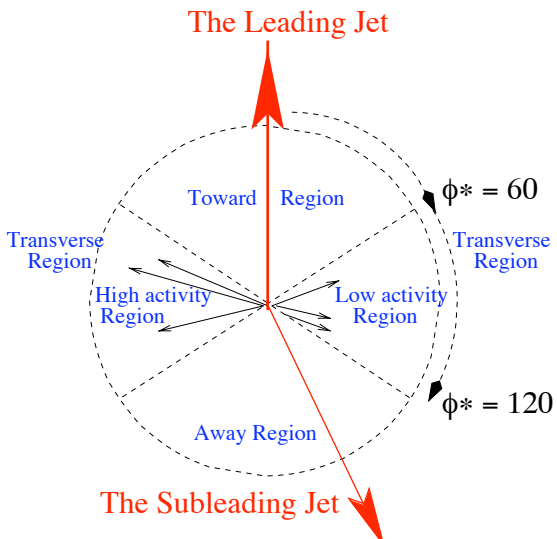

Figure 2: Definition of the four azimuthal regions.

The analysis procedure, inspired by the CDF collaboration [6], is the following:

Four regions in the azimuthal angle, $\phi$, were defined with respect to the leading jet as indicated in figure 2. The leading jet defines the azimuthal angle, $\phi=0$. The region $|\phi|<60^{\circ}$ is defined as the toward region and is expected to contain all particles from the leading jet. The away region is defined by $|\phi|>120^{\circ}$ which often contains the second leading jet and most of its particles. In the transverse regions, $60^{\circ}<|\phi|<120^{\circ}$, the contribution from the primary collision is usually small and thus the effects from the UE should be most visible.

In the transverse regions, a high activity and a low activity region are defined event by event depending on which region contains the higher scalar sum of the transverse momentum of charged particles, $P_{T}^{\text {sum }}=$ $\sum_{i}^{\text {tracks }} P_{T}^{i}$. The high activity region is more affected by higher order QCD contributions than the low activity region by definition: if higher order radiation is emitted this will increase the $P_{T}^{\text {sum }}$ in that transverse hemisphere unlike the other one.

The average charged particle multiplicity, $\left\langle N_{\text {charged }}\right\rangle$, as a function of the transverse momentum of the leading jet, $P_{T}^{\text {Jet }_{1}}$, for the different azimuthal regions is shown in figures 3 - 

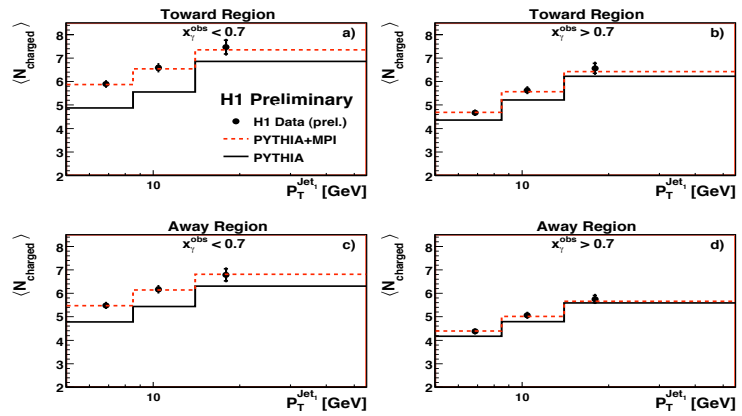

Figure 3: Average charged particle multiplicity as a function of the transverse momentum of the leading jet, $P_{T}^{J e t_{1}}$.
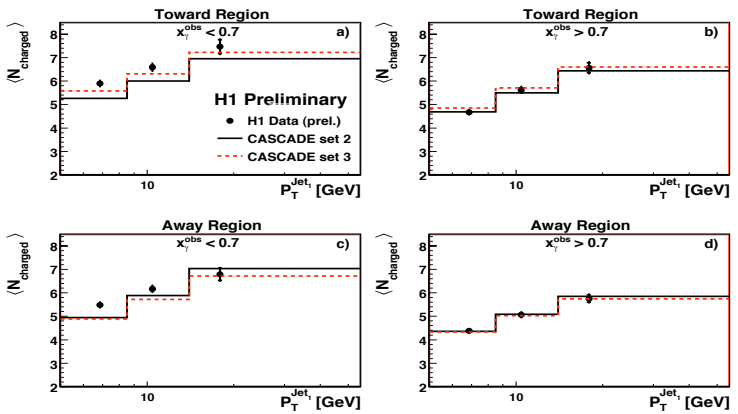

Figure 4: Average charged particle multiplicity as a function of the transverse momentum of the leading jet, $P_{T}^{J e t_{1}}$.

6. The measurement is performed for resolved and direct photon enriched events, i.e. $x_{\gamma}^{o b s}<$ 0.7 and $x_{\gamma}^{o b s}>0.7$, respectively.

The $\left\langle N_{\text {charged }}\right\rangle$ distributions are corrected to the level of charged stable hadrons using an iterative Bayes unfolding method (see [7]). They are compared to two MC predictions: Pythia [8] and CASCADE [9, 10]. Both implement matrix elements at leading order in $\alpha_{s}$. The matrix elements are supplemented with initial and final state radiation according to the DGLAP evolution equations in PYTHIA and the ones of CCFM in CASCADE . In PYTHIA a model of MPI is available for ep collisions. CASCADE uses unintegrated gluon density functions (updf) and off-shell matrix elements. It does not include the resolved component of the photon and has not model for MPI implemented. In PYTHIA the CTEQ $6 \mathrm{~L}$ [11] parton density function was used while in CASCADE set2 and set3 [12] were used.

In the toward and away regions $\left\langle N_{\text {charged }}\right\rangle$ increases with $P_{T}^{\text {Jet }_{1}}$ by about $30 \%$ from the lowest to the highest $P_{T}^{J e t_{1}}$ bin. On the contrary, in the transverse regions the multiplicity tends to decrease although the effect is much weaker. In the toward regions the particle multiplicity is slightly higher than in the away regions but in the transverse high activity regions the multiplicity is much higher than in the low activity regions. The multiplicity is higher for resolved enriched than for direct enriched events.

In figures 3 and 4 the data are compared to different MC predictions in the toward and away regions. The PYTHIA MC describes the data quite well if contributions from MPI are included in the simulation (figure 3 ). The contributions from MPI decrease as $P_{T}^{J e t_{1}}$ grows according to this model. The CASCADE MC describes the data fairly well. For direct 

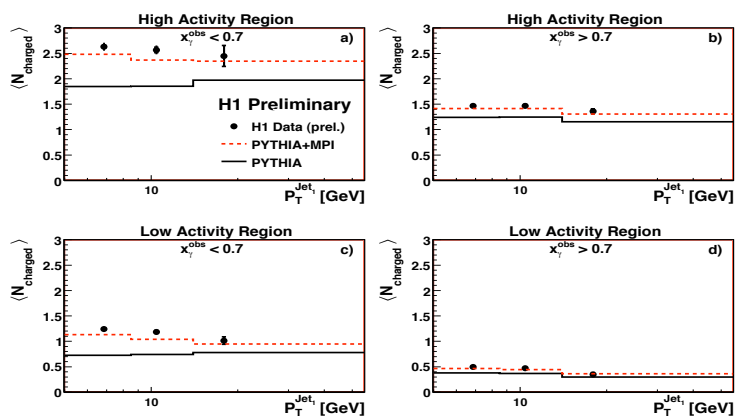

Figure 5: Average charged particle multiplicity multiplicity as a function of the transverse momentum of the leading jet, $P_{T}^{J e t_{1}}$.
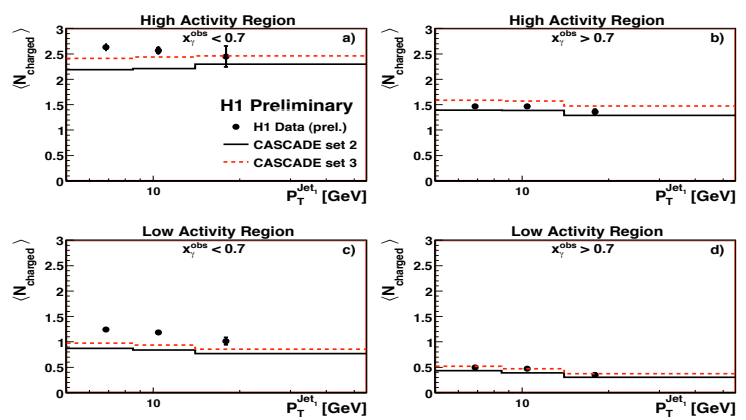

Figure 6: Average charged particle multiplicity multiplicity as a function of the transverse momentum of the leading jet, $P_{T}^{J e t_{1}}$.

enhanced events, $x_{\gamma}^{o b s}>0.7$, CASCADE describes the data perfectly. For resolved enhanced events, $x_{\gamma}^{\text {obs }}<0.7$, however, the predicted multiplicity is lower than in data, especially at low $P_{T}^{J e t_{1}}$.

Figures 5 and 6 show a comparison between data and MC predictions in the transverse regions. Like in the toward and away regions, including MPI improves the description of the data in all bins for PYTHIA ${ }^{\mathrm{a}}$. In both $x_{\gamma}^{\text {obs }}>0.7$ transverse regions ( $\mathrm{b}$ and $\mathrm{d}$ ) PYTHIA + MPI and CASCADE describe the data well. However, they somewhat underestimate the data in the resolved enriched transverse regions. Here, the shape predicted by PYTHIA + MPI follows the data distribution, although the absolute value of the multiplicity is slightly too low. CASCADE predicts an even lower multiplicity in these regions but it is much better than PYTHIA without MPI, although CASCADE does not include a resolved component and any MPI model. The description of CASCADE is better in the high activity region, where higher order corrections are more important, than in the low activity region, which is expected to be most sensitive to MPI. These discrepancies decrease with increasing $P_{T}^{J^{J} t_{1}}$.

\subsection{Conclusion}

The average charged particle multiplicity in dijet photoproduction has been measured as a function of $P_{T}^{\text {Jet }_{1}}$ in four regions of the azimuthal angle $\phi$ : the toward, away, transverse high and low activity regions. The data have been investigated for enhanced photon point-like

a PYTHIA describes the data only when including MPI. For more details see $[3,4]$ 
interactions with the proton events and enhanced photon resolved events. The data have been compared to predictions of the PYTHIA and CASCADE MC generators.

PYTHIA without MPI does not produce enough particles, especially at low $x_{\gamma}^{\text {obs }}$ and in the transverse regions. Including MPI leads to a good description of the data.

CASCADE provides a good description of the data in the high $x_{\gamma}^{o b s}$ regions. In the low $x_{\gamma}^{o b s}$ regions it produces too few particles, especially in the low activity region.

CASCADE describes the data better than PYTHIA without MPI both at low $x_{\gamma}^{\text {obs }}$ and at high $x_{\gamma}^{\text {obs }}$, where contributions from MPI are smaller. The discrepancies of CASCADE with the data in the high activity region are smaller than in the low activity region, the former is expected to be more sensitive to higher orders and the latter to MPI.

It has been shown that the data presented here is sensitive to the different models and provides important information about the parton shower approaches and MPI. Following the strategy of this analysis, new observables could provide additional information [4].

\section{Acknowledgments}

This work has been partially supported by the Spanish Consolider-Ingenio 2010 Programme CPAN (CSD2007-00042).

\section{References}

[1] S. Aid et al. Jets and energy flow in photon - proton collisions at HERA. Z. Phys., C70:17-30, 1996.

[2] S. Alekhin et al. HERA and the LHC - A workshop on the implications of HERA for LHC physics: Proceedings Part A. 2005.

[3] Ll. Marti. Study of Multiple Interactions in Photoproduction at HERA. H1-Preliminary-08-036.

[4] Lluis Marti Magro. Multiple parton interactions in photoproduction at HERA- H1. DESY-THESIS2009-007.

[5] S. Catani, Yuri L. Dokshitzer, M. H. Seymour, and B. R. Webber. Longitudinally invariant K(t) clustering algorithms for hadron hadron collisions. Nucl. Phys., B406:187-224, 1993.

[6] Darin E. Acosta et al. Soft and hard interactions in $p \bar{p}$ collisions at $\sqrt{s}=1800-\mathrm{GeV}$ and $630-\mathrm{GeV}$. Phys. Rev., D65:072005, 2002.

[7] G. D'Agostini. A Multidimensional unfolding method based on Bayes' theorem. Nucl. Instrum. Meth., A362:487-498, 1995.

[8] Torbjorn Sjostrand, Stephen Mrenna, and Peter Skands. PYTHIA 6.4 Physics and Manual. JHEP, 05:026, 2006

[9] H. Jung and G. P. Salam. Hadronic final state predictions from CCFM: The hadron- level Monte Carlo generator CASCADE. Eur. Phys. J., C19:351-360, 2001.

[10] H. Jung. The CCFM Monte Carlo generator CASCADE. Comput. Phys. Commun., 143:100-111, 2002.

[11] J. Pumplin et al. New generation of parton distributions with uncertainties from global QCD analysis. JHEP, 07:012, 2002.

[12] M. Hansson and H. Jung. Status of CCFM: Un-integrated gluon densities. 2003. 